\title{
Edouard Zarifian
}

\author{
Pascal-Henri KELLER*
}

*Professeur de psychologie Clinique et Psychopathologie, Psychanalyste, UFR de Psychologie, Université de Poitiers, 40, avenue Recteur Pineau, 86000 Poitiers, France. pascal.keller@ univ-poitiers.fr

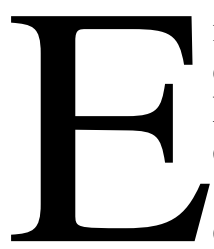

$\mathrm{n}$ se définissant lui-même comme un "tisseur de liens », Edouard Zarifian ne doutait pas que ces liens inlassablement tissés lui survivent; mais une fois établis, pouvait-il se douter de l'intensité avec laquelle ils demeureraient vivants et féconds ? Après sa mort, intervenue le 20 février dernier, ceux qui l'ont connu et aimé comprennent à quel point ce «tissage » les a rapprochés, combien il les aide à supporter son absence et à vivre désormais sans lui. Par ailleurs, s'il savait tisser des liens entre les personnes, il savait aussi jeter des ponts entre les communautés les plus diverses, qu'il s'agisse de disciplines scientifiques ou de milieux sociaux hétérogènes. Et tous les groupes humains où sa générosité a trouvé un écho peuvent en témoigner aujourd'hui. Aimante et unie, sa propre famille a été le premier lieu où, avec sa femme, sa fille et son fils, il a puisé ce dont il avait besoin pour accomplir son œuvre. Au-delà de cette faculté relationnelle hors du commun, son existence a été guidée par un engagement passionné : l'écoute et le soin destinés aux personnes frappées par la souffrance psychique et la maladie mentale. Dès le début de sa carrière de psychiatre, il a souhaité entreprendre une psychanalyse, ce qui ne l'a pas empêché plus tard de fonder l'Association française de psychiatrie biologique (dans les années 1970): ses efforts pour apaiser les tourments psychiques se sont toujours déployés sans préjugé aucun. Avant tout préoccupé par l'humain, il a délaissé la modélisation animale et les essais randomisés où la psychiatrie biologique s'est peu à peu enfermée, en grande partie sous la contrainte des laboratoires pharmaceutiques. Considérant cette méthodologie de la recherche en psychiatrie comme inappropriée à la réalité cli- nique, il s'est malgré tout intéressé à l'imagerie cérébrale, réussissant le cas échéant à doter de ce matériel coûteux certaines institutions où il travaillait. Il pensait sur ces questions et agissait en fonction du principe selon lequel le cerveau est la condition nécessaire à l'avènement de la pensée, sans être pour autant une condition suffisante.

Le prix de l'humanisme médical reçu il y a presque 20 ans pour son livre Les jardiniers de la folie tient au fait qu'il y préconisait, non pas l'éradication des maladies mentales sur le modèle pasteurien, mais le renouvellement de l'intérêt de tous pour ces souffrances singulières, dont seule, la parole porte véritablement témoignage. Il aurait voulu que chaque psychiatre, chaque soignant en psychiatrie, se laisse guider par cette phrase de Boris Vian : « Ce qui m'intéresse, ce n'est pas le bonheur de tous les hommes, c'est celui de chacun ». Fondés sur cette conception profondément humaniste de la souffrance psychique, son attachement et sa fidélité à la psychanalyse ne se seront jamais démentis. En 2004, il s'est rapproché de la recherche en psychopathologie clinique, rejoignant le Séminaire inter-universitaire européen d'étude et de recherche en psychopathologie et psychanalyse (SIUEERPP) fondé par Pierre Fédida et dirigé par Roland Gori. Fin 2006, seule la maladie l'a empêché d'être présent aux 80 ans de la Société psychanalytique de Paris (SPP), où il souhaitait parler des apports respectifs entre psychiatres cliniciens et psychanalystes.

Auteur inépuisable, il laisse une œuvre considérable où vont aujourd'hui puiser, non seulement les praticiens animés d'une même passion pour l'humain, mais aussi le grand public, auquel il s'est toujours adressé de façon accessible. Considérant que la psychiatrie se devait d'être au service des malades et non l'inverse, 
il s'est opposé à la réification des symptômes psychiques imposée par le DSM-IV, faisant valoir que seule, une approche personnalisée de ces «symptômes » permet d'en révéler, pour mieux l'explorer, l'inhérente dimension subjective. S'il souhaitait réserver l'usage du DSM à la seule recherche psychiatrique, il savait aussi, lorsque c'était nécessaire, recourir aux médicaments psychotropes et les prescrire le moment venu; il ne s'est pourtant jamais interdit d'en dénoncer l'usage abusif, en France tout particulièrement. Loin de fonder ses critiques sur une idéologie " anti-médicament», Edouard Zarifian a toujours pris soin d'en établir le bien-fondé à partir d'études rigoureuses et de données incontestables. En lui confiant la mission d'évaluer l'usage de ces médicaments en France par rapport aux autres pays européens, le ministère de la santé reconnaissait de facto sa compétence et son intégrité dans ce domaine. À partir du rapport rédigé au terme de sa mission, Odile Jacob lui a proposé de publier un livre : Le prix du bien-être. Il y démontre que si l'on connaît bien les chiffres des prescriptions de médicaments psychotropes - et sur ce plan, la France bat tous les pays européens - il est impossible d'avoir la moindre certitude quant à leur consommation. Contrairement à ceux qui permettent de traiter les maladies organiques, les médicaments du psychisme ont une particularité venant de leur mode de consommation qui, soumise à l'appréciation subjective du patient, demeure imprévisible.

Quelques semaines avant sa mort, il parlait des multiples projets qu'il avait en tête. S'il voulait poursuivre avec sa femme les visites dans le bordelais qu'ils affectionnaient tous les deux ainsi que sa collaboration avec le monde du champagne, il avait non seulement l'intention d'approfondir la peinture et de passer davantage de temps devant son chevalet, mais souhaitait aussi se replonger dans la poésie espagnole et la littérature. Par ailleurs, il avait toujours envie d'écrire et voulait continuer, de cette manière, à transmettre l'immense savoir accumulé tout au long de sa vie. Sur ce plan, deux projets d'ouvrages lui tenaient particulièrement à cœur. Explorant sa conception du psychisme en tant qu'expression singulière de l'identité humaine, le premier devait en présenter la «bulle» comme une traduction métaphorique: image de la solitude et de l'isolement, la bulle est aussi l'expression de l'effervescence collective et du pétillement. Il désirait se laisser entraîner par cette métaphore dans une vaste réflexion sur la condition humaine, issue de son atypique et inventive trajectoire personnelle. Davantage polémique, le second ouvrage avait pour objectif de contredire, voire d'entraver, les discours fondés sur l'imposture ; impitoyable sur ce que la littérature scientifique produit de mystifications et d'abus de langage, il tenait à en démasquer les procédés. Dans ce projet, il voulait s'en prendre à toutes les formes de scientisme pour mieux rendre à la science la noblesse de sa démarche, surtout quand elle se donne pour mission d'étudier les avatars du psychisme humain. Ces ouvrages sont dans l'état où il les a laissé : de simples ébauches. Aujourd'hui, seule une volonté comparable à la sienne et farouchement décidée à ne plus s'en laisser compter par les modes, les idéologies ou les imposteurs, pourrait tenter de faire exister ces livres, après en avoir retrouvé l'intention première.

Pour terminer cet hommage au grand psychiatre humaniste qui vient de disparaître, un dernier mot sur son principal titre de gloire. Évoquant sa longue carrière, il se disait fier de constater, aussi loin qu'il se souvienne, qu'aucun de ses patients n'était mort par suicide. Quel psychiatre n'en éprouverait de la fierté ? Le dernier livre d'Edouard Zarifian s'intitule Le goût de vivre: plus qu'un programme ou un acte de foi, ce titre désigne en réalité ce qu'il a passé sa vie à vouloir partager.

\section{Communiqué}

$70^{\mathrm{e}}$ anniversaire de la SFPEADA

\section{Société Française de Psychiatrie de l'Enfant et de l'adolescent et Disciplines Associées $1^{\text {er }}$ et 2 juin 2007}

Maison de la Chimie, Paris, France

Enfants d'ailleurs - Vivre les différences

Renseignements

BCA - Tél : 0141066770 - Email : contact@b-c-a.fr 